\title{
Can We Estimate Short- and Intermediate-term Survival in Patients Undergoing Surgery for Metastatic Bone Disease?
}

\author{
Jonathan A. Forsberg MD, PhD, Rikard Wedin MD, PhD, Patrick J. Boland MD, \\ John H. Healey MD
}

Received: 23 May 2016/ Accepted: 21 November 2016/Published online: 1 December 2016

(C) The Association of Bone and Joint Surgeons \& 2016

\begin{abstract}
Background Objective means of estimating survival can be used to guide surgical decision-making and to risk-stratify patients for clinical trials. Although a free, online tool (www. pathfx.org) can estimate 3- and 12-month survival, recent work, including a survey of the Musculoskeletal Tumor Society, indicated that estimates at 1 and 6 months after surgery also would be helpful. Longer estimates help justify the need for more durable and expensive reconstructive
\end{abstract}

One of the authors $(\mathrm{JHH})$ received funding from the Maynard Limb Preservation Fund.

Research at Memorial Sloan Kettering Cancer Center is supported in part by a Cancer Center Support Grant from the U.S. National Institutes of Health/National Cancer Institute (\#P30 CA008748). All ICMJE Conflict of Interest Forms for authors and Clinical Orthopaedics and Related Research ${ }^{\mathbb{R}}$ editors and board members are on file with the publication and can be viewed on request.

Clinical Orthopaedics and Related Research ${ }^{\mathbb{R}}$ neither advocates nor endorses the use of any treatment, drug, or device. Readers are encouraged to always seek additional information, including FDAapproval status, of any drug or device prior to clinical use.

Each author certifies that his or her institution approved the human protocol for this investigation, that all investigations were conducted in conformity with ethical principles of research, and that informed consent for participation in the study was obtained.

This work was performed at the Naval Medical Research Center, Silver Spring, MD, USA.

The views expressed in this article are those of the authors and do not necessarily reflect the official policy or position of the Department of the Navy, the Department of Defense, nor the U.S. Government. Research activities leading to the development of this abstract were approved by the local Institutional Review Board (NMRC.2014.0009 and IRBNet 392350) in compliance with all applicable regulations governing the protection of human subjects. One of the authors (JAF) is a military service member. This work was supported/funded by work-unit number HU0001-14-1-0010 and was prepared as part of his official duties. Title 17 U.S.C. $\$ 105$ provides that "Copyright protection under this title is not available for any work of the United States Government". Title 17 U.S.C. $\$ 101$ defines a U.S. government options, and very short estimates could help identify those who will not survive 1 month and should not undergo surgery. Thereby, an important use of this tool would be to help avoid unsuccessful and expensive surgery during the last month of life.

Questions/Purposes We seek to provide a reliable, objective means of estimating survival in patients with metastatic bone disease. After generating models to derive 1- and 6-month survival estimates, we determined suitability for clinical use by applying receiver operator

work as a work prepared by a military service member or employee of the U.S. Government as part of that person's official duties. We certify that the document represents valid work; that if we used information derived from another source, we obtained all necessary approvals to use it and made appropriate acknowledgements in the document; and we take public responsibility for it.

\section{J. A. Forsberg (}

Regenerative Medicine, Naval Medical Research Center, 503

Robert Grant Avenue, Silver Spring, MD 20910, USA

e-mail: jaforsberg@me.com

\section{J. A. Forsberg}

Uniformed Services University-Walter Reed Department of Surgery, Bethesda, MD, USA

J. A. Forsberg, R. Wedin

Section of Orthopaedics and Sports Medicine, Department of Molecular Medicine and Surgery, Karolinska Institute,

Karolinska University Hospital, Stockholm, Sweden

P. J. Boland, J. H. Healey

Orthopaedic Surgery Service, Department of Surgery, Memorial Sloan-Kettering Cancer Center and Weill College of Medicine, Cornell University, New York, NY, USA 
characteristic $(\mathrm{ROC})$ (area under the curve $[\mathrm{AUC}]>0.7$ ) and decision curve analysis (DCA), which determines whether using PATHFx can improve outcomes, but also discerns in which kinds of patients PATHFx should not be used.

Methods We used two, existing, skeletal metastasis registries chosen for their quality and availability. Data from Memorial Sloan-Kettering Cancer Center (training set, $\mathrm{n}=189$ ) was used to develop two Bayesian Belief Networks trained to estimate the likelihood of survival at 1 and 6 months after surgery. Next, data from eight major referral centers across Scandinavia $(n=815)$ served as the external validation set-that is, as a means to test model performance in a different patient population. The diversity of the data between the training set from Memorial SloanKettering Cancer Center and the Scandinavian external validation set is important to help ensure the models are applicable to patients in various settings with differing demographics and treatment philosophies. We considered disease-specific, laboratory, and demographic information, and the surgeon's estimate of survival. For each model, we calculated the area under the ROC curve (AUC) as a metric of discriminatory ability and the Net Benefit using DCA to determine whether the models were suitable for clinical use.

Results On external validation, the AUC for the 1- and 6month models were 0.76 (95\% CI, 0.72-0.80) and 0.76 (95\% CI, 0.73-0.79), respectively. The models conferred a positive net benefit on DCA, indicating each could be used rather than assume all patients or no patients would survive greater than 1 or 6 months, respectively.

Conclusions Decision analysis confirms that the 1- and 6month Bayesian models are suitable for clinical use.

Clinical Relevance These data support upgrading www. pathfx.org with the algorithms described above, which is designed to guide surgical decision-making, and function as a risk stratification method in support of clinical trials. This updating has been done, so now surgeons may use any web browser to generate survival estimates at 1, 3, 6, and 12 months after surgery, at no cost. Just as short estimates of survival help justify palliative therapy or less-invasive approaches to stabilization, more favorable survival estimates at 6 or 12 months are used to justify more durable, complicated, and expensive reconstructive options.

\section{Introduction}

Survival estimates are important when treating patients with metastatic bone disease to help set patient, family, and physician expectations. For orthopaedic surgeons, objective means of estimating life expectancy can be used to guide surgical decision-making, thereby helping to identify which patients may benefit from surgery and which may require more durable reconstructive options. In addition, validated means by which to estimate longevity may be used to risk-stratify patients before enrollment in clinical trials. Unfortunately, physician estimates alone are notoriously inaccurate $[7,8]$, which stimulated the development of more objective means of estimating life expectancy in this terminal but not necessarily terminally ill patient population.

One application designed for use in patients with metastatic bone disease is PATHFx [11]. Introduced in 2011 [4], this validated clinical decision-support tool [6] uses clinical and physiologic variables to generate the probability of survival at 3 and 12 months after orthopaedic surgery. It is available free at www.pathfx.org. These times were chosen by expert opinion as a means to help surgeons determine which patients would benefit from surgery (probability of survival at 3 months) and whether a more-durable implant may be necessary (probability of survival at 12 months). However, given the relatively broad array of treatment options for patients with symptomatic bone metastases, survival estimates at other times may be preferred in certain situations. For instance, those considering palliative options for metastatic lesions may seek to estimate very short (1-month) life expectancy [10]. In addition, more-favorable estimates of 1-6 months may justify less-invasive approaches to stabilization such as intramedullary fixation (Fig. 1A). Finally, a survey of Musculoskeletal Tumor Society members [12] indicated that the probability of survival at 6 months postsurgery would be helpful in determining whether to choose a moredurable reconstruction option involving conventional or modular "tumor" prostheses, specifically for lesions involving the peritrochanteric or subtrochanteric femur (Fig. 1D). Similarly, for patients with favorable estimates at 12 months postsurgery, the evidence supporting the need for more-durable implants becomes stronger.

Although various statistical techniques could be used to estimate life expectancy, a Bayesian approach using probabilistic theory appears well suited to this clinical application, in part because it can accommodate uncertainty by functioning in the presence of incomplete information, common in the clinical setting. In addition, Bayesian networks are capable of estimating the likelihood of rare events, which may be useful when attempting to estimate very short $(<1$ month) life expectancy in a population of surgical candidates. The original Bayesian Belief Networks that we used to estimate 3- and 12-month survival have been externally validated twice $[6,11]$ and possess favorable characteristics on decision curve analysis (DCA) [14], indicating that the models are suitable for clinical use. As such, it is reasonable to determine whether 1-month and 6-month survival could be estimated using 

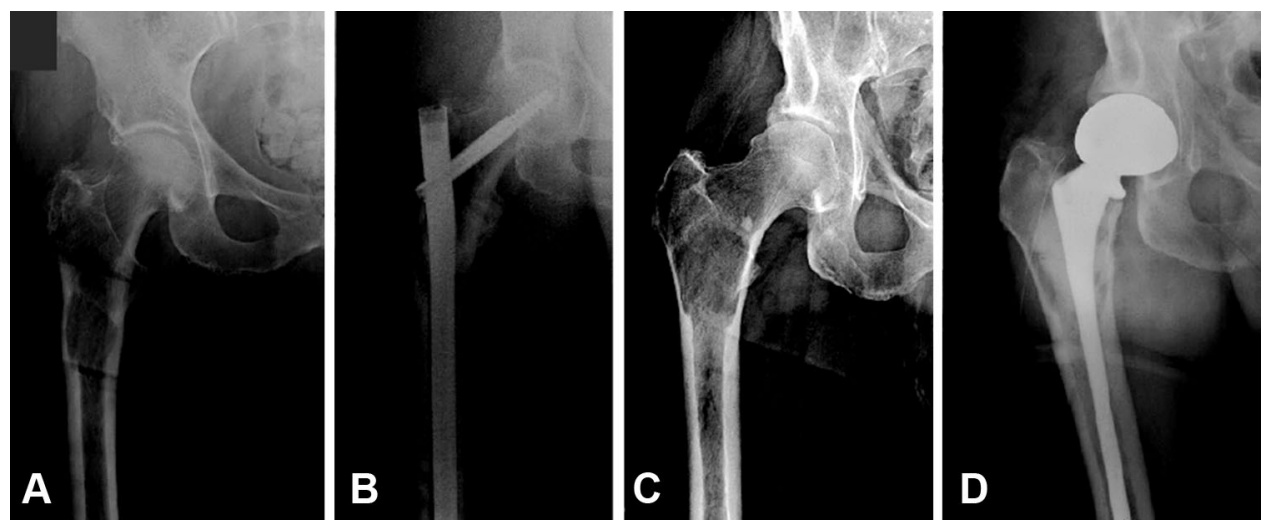

Fig. 1A-D Two implant options for patients with impending pathologic proximal femur fractures are shown. Relatively sick patients with high likelihood of perioperative complications and survival estimates less than 6 months may benefit from intramedullary stabilization. (A) Preoperative and (B) postoperative radiographs are shown. Healthier patients who have a lower likelihood of

similar techniques. In doing so, we respond to treatment philosophies preferred by the orthopaedic oncology community [12] and provide clinicians with an objective means by which to characterize each patient's life expectancy. If successful, www.pathfx.org could be upgraded to provide the user with a comprehensive, no-cost, assessment of each patient's survival trajectory, by estimating 1-, 3-, 6-, and 12-month postoperative survival.

With this in mind, we developed two Bayesian models capable of estimating the likelihood of 1- and 6-month survival in patients undergoing surgery for metastatic bone disease. On external validation, we performed receiver operator characteristic (ROC) and DCA [14] to determine whether each model was suitable for clinical use.

\section{Patients and Methods}

After local institutional review board approval, we retrospectively reviewed our longitudinally maintained database and identified the records of 189 patients who underwent surgery for skeletal metastases at Memorial Sloan-Kettering Cancer Center (MSKCC) between 1999 and 2003 (MSKCC training set). Each record contained 15 variables and sufficient information to establish survival at 6 months postsurgery (yes or no) in $100 \%$ of records. No patient was lost to followup. Other recorded variables included age at surgery, race, sex, primary oncologic diagnosis, indication for surgery (impending or complete pathologic fracture), number of bone metastases (solitary or multiple), presence or absence of visceral (organ) metastases, estimated glomerular filtration rate $\left(\mathrm{mL} /\right.$ minute $\left./ 1.73 \mathrm{~m}^{2}\right)$, serum calcium concentration $(\mathrm{mg} / \mathrm{dL})$, serum albumin concentration $(\mathrm{g} / \mathrm{dL})$, presence or absence of lymph node perioperative complications and more favorable survival estimates of greater than 6 months may require more durable implants, such as the prosthesis shown here. (C) Preoperative and (D) postoperative radiographs are shown. Patients with very short life expectancies of less than 1 month may be candidates for palliative radiotherapy, without surgery.

metastases, prior chemotherapy (yes or no), preoperative hemoglobin $(\mathrm{g} / \mathrm{dL}$; on admission, before transfusion, if applicable), absolute lymphocyte count $(\mathrm{K} / \mathrm{mL})$, and the senior surgeons' (JHH and PJB) estimates of survival (postoperatively in months).

Including the surgeon's estimate - a subjective assessment-may seem controversial. However, doing so allows surgeons with considerable expertise to provide a weighted estimate of survival that can be used in conjunction with the other, more-objective, features contained in PATHFx. Although the surgeon's estimate contains many important subjective features that cannot be quantified, it does demand a certain level of experience. If surgeons are unsure regarding whether an estimate is appropriate, he or she should select "unknown." Doing so will maintain accuracy of the model, while not introducing undue bias, as shown in two prior external validation studies $[6,11]$.

We used these data to develop two Bayesian models in a manner previously described [5] using commercially available machine-learning software (FasterAnalytics ${ }^{\mathrm{TM}}$; DecisionQ, Washington, DC, USA). In brief, all 15 variables were considered as candidate features for inclusion in the models. Prior distributions, that is, the values each variable is likely to assume under various circumstances, was learned from the MSKCC training set and not specified a priori. We then generated two models containing the outcomes, 1-month or 6-month survival (yes or no), and generated calibration curves that plotted predicted risk against actual risk to assess the accuracy of model predictions.

We next used data from eight major referral centers across Scandinavia $(\mathrm{n}=815)$ to perform external validation (Scandinavian external validation set). We chose this registry because it is well-characterized, and was used to 
externally validate the original 3- and 12-month PATHFx models [6]. In general, models are not suitable for clinical practice until external validation is performed. The Scandinavian external validation set provided a means to test model performance by requiring the 1- and 6-month models to estimate survival in each of the 815 "unknowns." The diversity of the data between the MSKCC training set and the Scandinavian external validation set is important to help ensure the models can be used to guide treatment in various settings with differing patient populations, demographics, and treatment philosophies. Each record contained the preoperative features required to validate the 1-month and 6-month models. Followup was sufficient to establish postoperative survival at 6 months in $100 \%$ of records. Ethical approval was not required before using these deidentified registry data. Importantly, the senior surgeons' estimates of survival were not contained in the external validation data set. Choosing a validation set without the surgeon's estimate was intentional and helps ensure these models are applicable to centers that may lack expertise in estimating life expectancy for this patient population.

Using the Scandinavian external validation set, we determined the discriminatory ability of each model in estimating the likelihood of 1- or 6-month survival by sensitivity, specificity, and ROC analysis [2]. A minimum ROC area under the curve (AUC) of 0.7 was considered acceptable and was specified a priori.

Measures of accuracy and discrimination alone cannot determine whether the models are suitable for clinical use. Other methods that weigh the consequences of false-positive and false-negative result models are also necessary. To accomplish this, we used DCA [14], an analytic technique that helps quantify the consequences of under- and/or overtreatment of the disease. When constructing decision curves, we assumed surgical decisions would be based strictly on the output of each model. For instance, the decision to offer palliative care or surgery would be based on the likelihood of 1-month survival, and the choice of implant (intramedullary nail vs endoprostheses) would be based on the output of the 6-month model. In each setting, the consequences of undertreatment differ from those associated with overtreatment, which serves as the basis for decision analysis. When interpreting the curves, surgeons must consider his or her "threshold probability," or the point of equipoise at which he or she is indecisive regarding which treatment is best. Surgeons usually have a low threshold for treatment when dealing with healthy patients, and higher thresholds with sicker patients. Because thresholds vary by surgeon, patient, and situation, it is important to choose a decision analytic technique that allows one to evaluate one or more models across a range of clinically relevant thresholds. Importantly, the decision curves do not estimate the likelihood of survival (the PATHFx models serve this purpose), but rather help determine which model(s) should and should not be used in certain clinical situations represented by a given surgeon's threshold. Wherever possible, we included information recommended by the Transparent Reporting of a Multivariable Prediction Model for Individual Prognosis or Diagnosis (TRIPOD) [1]. In addition to these, we believe decision analytic techniques, such as those used in this study, are important requirements for any model destined for the clinical setting [3].

\section{Results}

The Bayesian models successfully estimated 1- and 6month survival. The graphic structure of each model helps one understand the relationships between features (Fig. 2), and by inspection of the calibration plots, both were

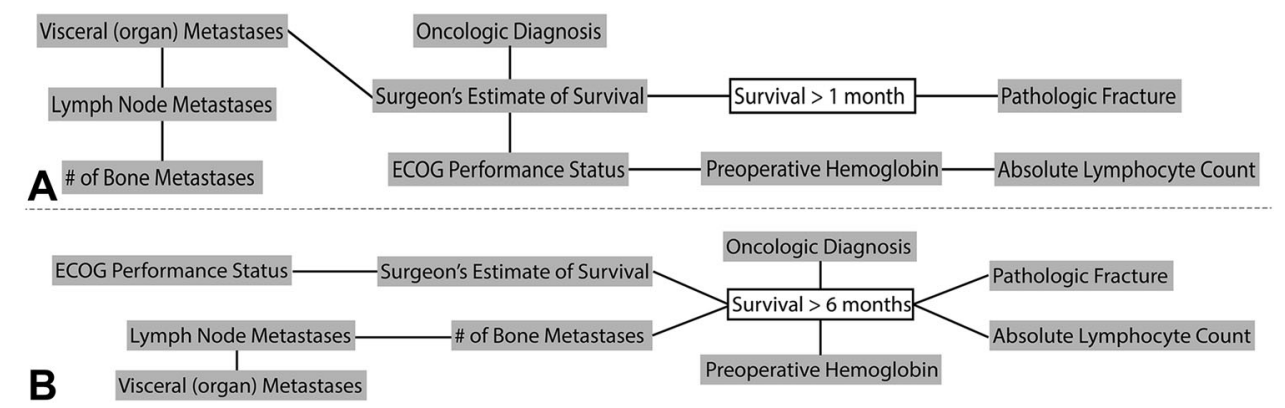

Fig. 2A-B The Bayesian Belief Network structure of the (A) 1month and (B) 6-month models are shown. There are two first-degree associates of 1-month survival. These are the features that are most highly related to the outcome of interest and include the senior surgeon's estimate of survival and the presence of a completed (as opposed to an impending) pathologic fracture. In comparison, there are six first-degree associates of 6-month survival, including preoperative hemoglobin, absolute lymphocyte count, oncologic diagnosis, presence of a completed pathologic fracture, the number of bone metastases, and the senior surgeon's estimate of survival. 
Fig. 3A-B The calibration curves show the agreement between observed outcomes and those predicted by the (A) 1-month and (B) 6-month PATHFx models. The shaded region depicts the $95 \% \mathrm{CI}$ of the predictions. Perfect calibration to the training data should overlie the $45^{\circ}$ dotted line. Both models are reasonably well calibrated to the MSKCC training data.

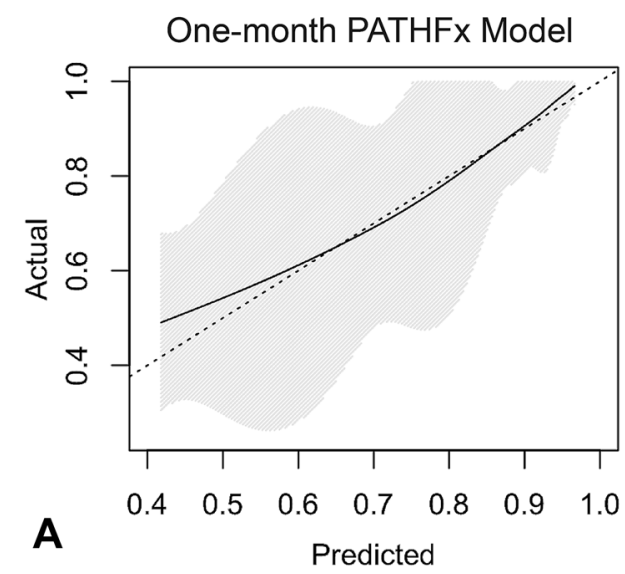

reasonably calibrated to the MSKCC training data (Fig. 3). In terms of model structure (Fig. 2), those features connected to one another are known as "first-degree associates," and are conditionally dependent - that is, the value of one feature can be represented in terms of one or more other features. First-degree associates are the features that are most closely related to and most influential in estimating the likelihood of a particular outcome. In fact, if the values for the first-degree associates are known, the values for the rest of the features are irrelevant. However, if a first-degree associate is missing, the models retain functionality and other features are used in its place. For instance, some surgeons may not choose to provide a surgeon's estimate of survival. For the 1-month model (Fig. 2A), the oncologic diagnosis, presence of visceral metastases, and Eastern Cooperative Oncology Group (ECOG) performance status serve as objective surrogates. For the 6-month model (Fig. 2B), ECOG performance status is used instead. As shown, there are two first-degree associates of 1-month survival (Fig. 2A) and six first-degree associates of 6-month survival (Fig. 2B). On external validation, the 1- and 6-month models correctly estimate survival in $80 \%$ and $60 \%$ records, respectively. For the 1month model, 11 of the 166 erroneous estimates (7\%) were underestimates and $155(93 \%)$ were overestimates. For the 6-month models, 100 of the 274 erroneous estimates $(37 \%)$ were underestimates and $174(64 \%)$ were overestimates. The AUC for the 1- and 6-month models were $0.76(95 \%$ CI, 0.72-0.80) and 0.76 (95\% CI, 0.73-0.79), respectively. Importantly, the models retained their discriminatory ability despite missing data, and notable differences in most continuous (Table 1) and categorical (Table 2) features. This highlights the unique advantages of Bayesian models to function in the presence of missing input data, even when confronted with differing patient populations and treatment philosophies.

DCA (Fig. 4) indicated that both models possess clinical utility. Net benefit is defined as a 1- or 6-month survivor who duly receives an operation and implant commensurate with his or her estimated survival. Importantly, decision curves do not recommend a course of treatment, but rather help determine whether PATHFx should be used to achieve better outcomes in various clinical situations [5]. To interpret the decision curves, a surgeon must first determine his or her threshold for treatment, known as the threshold probability. This is the point of equipoise between recommending surgery or nonoperative treatment, in the case of 1-month survival, or deciding between an intramedullary nail or prosthesis, in the case of 6-month survival. The threshold differs between surgeons and is patient-, tumor-, and situation-dependent. For example, some may require a $50 \%$ probability of 1-month survival before offering surgery, while others require only a $10 \%$. However, surgeons who are considering whether to offer surgery to a very sick patient, thought to be at high risk of having perioperative complications, may have a very high threshold. For example, if a surgeon's threshold exceeds $60 \%$, (eg, would offer surgery only if the probability of survival exceeded 60\%) (Fig. 4A), he or she should use PATHFx to derive 1-month survival estimates rather than assume the patient will or will not survive greater than 1 month. If a surgeon's threshold is less than $60 \%$, which can be the case in healthier patients thought to be at lower risk of perioperative complications, the PATHFx curves and the "assume all survive" curves are collinear. As such, he or she may either use PATHFx to derive a probability of 1-month survival, or simply proceed as if the patient will survive greater than 1 month.

Another important use of decision curves is to determine in which patients PATHFx should not be used. For instance, surgeons who are considering whether a more durable implant may be necessary by using the 6-month model, may have a high threshold for doing so in the setting of very sick patients. If his or her threshold for offering a prosthesis rather than an intramedullary nail exceeds $72 \%$ (Fig. 4B, arrow), surgeons may expect better outcomes by basing treatment decisions on the assumption that the patient will not survive greater than 6 months, rather than using the PATHFx tool. 
Table 1. Categorical variables contained in the training and validation sets

\begin{tabular}{|c|c|c|c|c|c|c|}
\hline \multirow[t]{2}{*}{ Feature } & \multicolumn{2}{|c|}{ Training set $(\mathrm{n}=189)$} & \multicolumn{3}{|c|}{ Validation set $(n=815)$} & \multirow[t]{2}{*}{$\mathrm{p}$ Value } \\
\hline & Number of patients & Percent & Number of patients & Percent & Percent missing & \\
\hline \multicolumn{7}{|l|}{ Gender } \\
\hline Male & 85 & 45 & 369 & 45 & 0 & 0.91 \\
\hline Female & 104 & 55 & 446 & 55 & & \\
\hline \multicolumn{7}{|c|}{ Diagnosis group } \\
\hline 1.0 & 52 & 27 & 173 & 21 & $<1$ & $<0.001^{*}$ \\
\hline 2.0 & 34 & 18 & 74 & 9 & & \\
\hline 3.0 & 103 & 55 & 567 & 70 & & \\
\hline \multicolumn{7}{|c|}{ Visceral metastases } \\
\hline Yes & 114 & 60 & 325 & 40 & 6 & $<0.001^{*}$ \\
\hline No & 75 & 40 & 441 & 54 & & \\
\hline \multicolumn{7}{|c|}{ Lymph node metastases } \\
\hline Yes & 36 & 19 & 169 & 21 & 62 & $<0.001 *$ \\
\hline No & 153 & 81 & 143 & 18 & & \\
\hline \multicolumn{7}{|c|}{ Number of bone metastases } \\
\hline Solitary & 55 & 29 & 123 & 15 & 3 & $<0.001 *$ \\
\hline Multiple & 134 & 71 & 666 & 82 & & \\
\hline \multicolumn{7}{|c|}{ Pathologic fracture } \\
\hline Complete & 84 & 44 & 614 & 75 & $<1$ & $<0.001 *$ \\
\hline Impending & 105 & 56 & 196 & 24 & & \\
\hline \multicolumn{7}{|c|}{ ECOG performance status } \\
\hline 0,1, or 2 & 93 & 49 & 558 & 68 & 0 & $<0.001 *$ \\
\hline 3 or 4 & 96 & 51 & 257 & 32 & & \\
\hline \multicolumn{7}{|c|}{ Survival $>1$ months } \\
\hline Yes & 173 & 92 & 622 & 87 & 0 & $<0.001 *$ \\
\hline No & 16 & 8 & 193 & 13 & & \\
\hline \multicolumn{7}{|c|}{ Survival $>6$ months } \\
\hline Yes & 111 & 59 & 372 & 46 & 0 & $0.001 *$ \\
\hline No & 78 & 41 & 443 & 54 & & \\
\hline
\end{tabular}

*Distributions are significantly different between the training and validation sets by the chi square method; Percent missing $=$ the proportion of unknown or missing data in the validation set; ECOG = Eastern Cooperative Oncology Group.

\section{Discussion}

The ability to derive objective, personalized survival estimates is important for all providers treating patients with metastatic bone disease. As the number of medical, surgical, and palliative therapies increase, there is a need to riskstratify patients in clinical and research settings. Toward this end, we successfully developed and externally validated two additional models able to estimate the likelihood of 1- and 6-month survival. We also showed that the models possess clinical utility, and added them to www. pathfx.org, which now is capable of estimating the likelihood of survival at $1,3,6$, and 12 months after surgery. In doing so, we acknowledge that surgeons may find it useful to obtain very-short (1 month) survival estimates in an effort to avoid expensive and unsuccessful surgery during the last month of life. In addition, favorable survival estimates of greater than 6 months may help justify moredurable, but complicated, reconstructive endoprosthetic options. By improving our ability to estimate each patient's postoperative survival trajectory, we ensure PATHFx remains current and relevant to the surgeon, who may now obtain a more complete representation of their patients' survival trajectory at www.pathfx.org. In addition we provide a validated tool that may be used for risk stratification before enrollment in clinical trials in this challenging and quite diverse patient population.

We note the following limitations. Given the data used in this study, it is probable that other statistical techniques could yield prognostic models. However, we have considerable experience using and applying Bayesian statistics. Moreover, the existing 3- and 12-month models 
Table 2. Continuous variables contained within the training and validation sets

\begin{tabular}{|c|c|c|c|c|}
\hline Feature & Training set $(n=189)$ & Validation set $(\mathrm{n}=815)$ & Percent missing & $\mathrm{p}$ Value \\
\hline \multicolumn{5}{|c|}{ Age at surgery (years) } \\
\hline Mean & 62 & 66 & \multirow[t]{4}{*}{0} & \multirow[t]{4}{*}{$<0.001 *$} \\
\hline SD & 14 & 13 & & \\
\hline Median & 63 & 67 & & \\
\hline IQR & 54,72 & 58,76 & & \\
\hline \multicolumn{5}{|c|}{ Hemoglobin (mg/dL) } \\
\hline Mean & 11.5 & 11.5 & \multirow[t]{4}{*}{0.6} & \multirow[t]{4}{*}{1.0} \\
\hline SD & 1.9 & 3.5 & & \\
\hline Median & 11.4 & 11.3 & & \\
\hline IQR & $10.1,12.9$ & $10.3,12.6$ & & \\
\hline \multicolumn{5}{|c|}{ Absolute lymphocyte count $(\mathrm{K} / \mu \mathrm{L})$} \\
\hline Mean & 1.2 & 1.2 & \multirow[t]{4}{*}{83.8} & \multirow[t]{4}{*}{0.48} \\
\hline SD & 1.3 & 0.74 & & \\
\hline Median & 1.0 & 1.2 & & \\
\hline IQR & $0.6,1.5$ & $0.8,1.6$ & & \\
\hline \multicolumn{5}{|c|}{ Surgeon's estimate of survival (months) } \\
\hline Mean & 10 & N/A & 100 & \multirow[t]{4}{*}{ N/A } \\
\hline SD & 9 & & & \\
\hline Median & 6 & & & \\
\hline IQR & 4,12 & & & \\
\hline
\end{tabular}

*Distributions are significantly different between training and validation sets by two-tailed Student's $\mathrm{t}$ test; Percent missing $=$ the proportion of unknown or missing data in the validation set; IQR = interquartile range; N/A = not applicable.

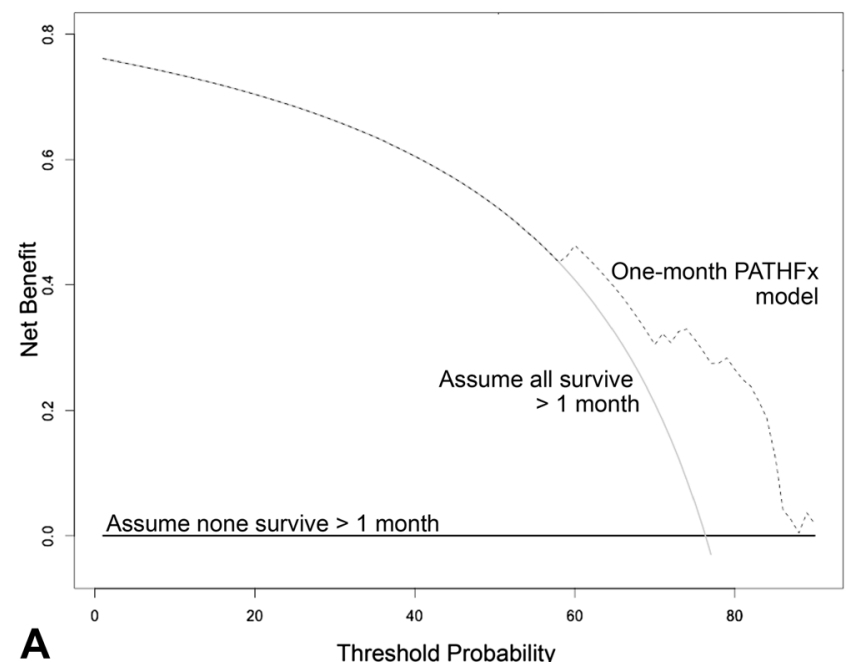

Fig. 4A-B The decision curves depict the net benefit of the (A) 1month and (B) 6-month models when applied to the Scandinavian external validation set. Net benefit is defined as a single patient who duly receives the correct treatment based on the model output. Each of the models could be used rather than assume all or none of the patients will survive greater than 1 or 6 months, respectively. However, surgeons requiring a high degree of probability of 6-month

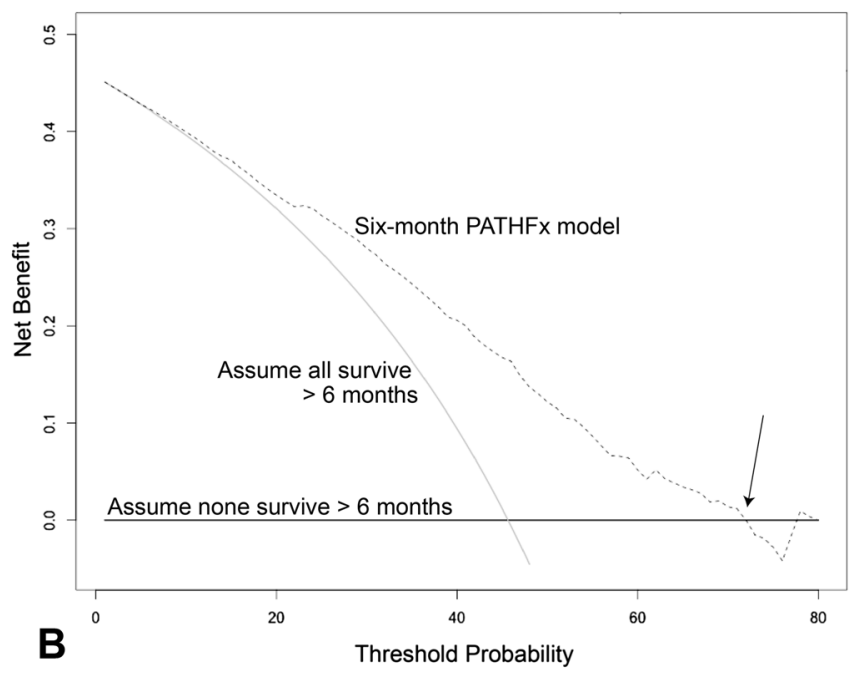

survival (B, arrow) before offering endoprostheses should base treatment decisions on the assumption that the patient will not survive greater than 6 months rather than use the PATHFx. This situation can be encountered with very sick patients for whom the risks of arthroplasty outweigh the benefits. In this case, surgeons may choose a less-invasive approach to stabilization, or palliative treatment, depending on the patient's 1- and 6-month survival estimates. 
have been externally validated twice $[6,11]$ and shown to possess clinical utility in other patient populations. Next, the data in the training and validation sets were derived from tertiary referral centers. As such, it is unknown if and how the resultant models would apply to the community setting. However, it was our intent to use an independent data set that did not contain the senior surgeons' estimates of survival to illustrate an important feature in Bayesian modeling - the ability to accommodate uncertainty in the clinical setting, and function in the presence of missing input data. Next, as with any modeling approach, overfitting can occur and produce overly optimistic results. This is typically the case when authors report internal validation statistics. However, by focusing our results on external validation, rather than internal validation, we suggest that the models retain accuracy when confronted by otherwise unrelated patient information and therefore, are not overfit to the training set. In addition, because the models were developed using data from patients who had undergone orthopaedic stabilization, only a small proportion $(8 \%$ of the MSKCC training set) survived less than 1 month. Nevertheless, Bayesian Belief Networks are able to estimate the likelihood of rare events, like very short-term survivors, by describing their unique characteristics and the relationships between features. Next, it is unclear whether PATHFx would be useful in nonsurgical settings to estimate life expectancy; however, we intend to validate them in patients undergoing palliative radiation therapy of symptomatic bone metastases. Finally, before using any clinical decision support tool, orthopaedic surgeons should

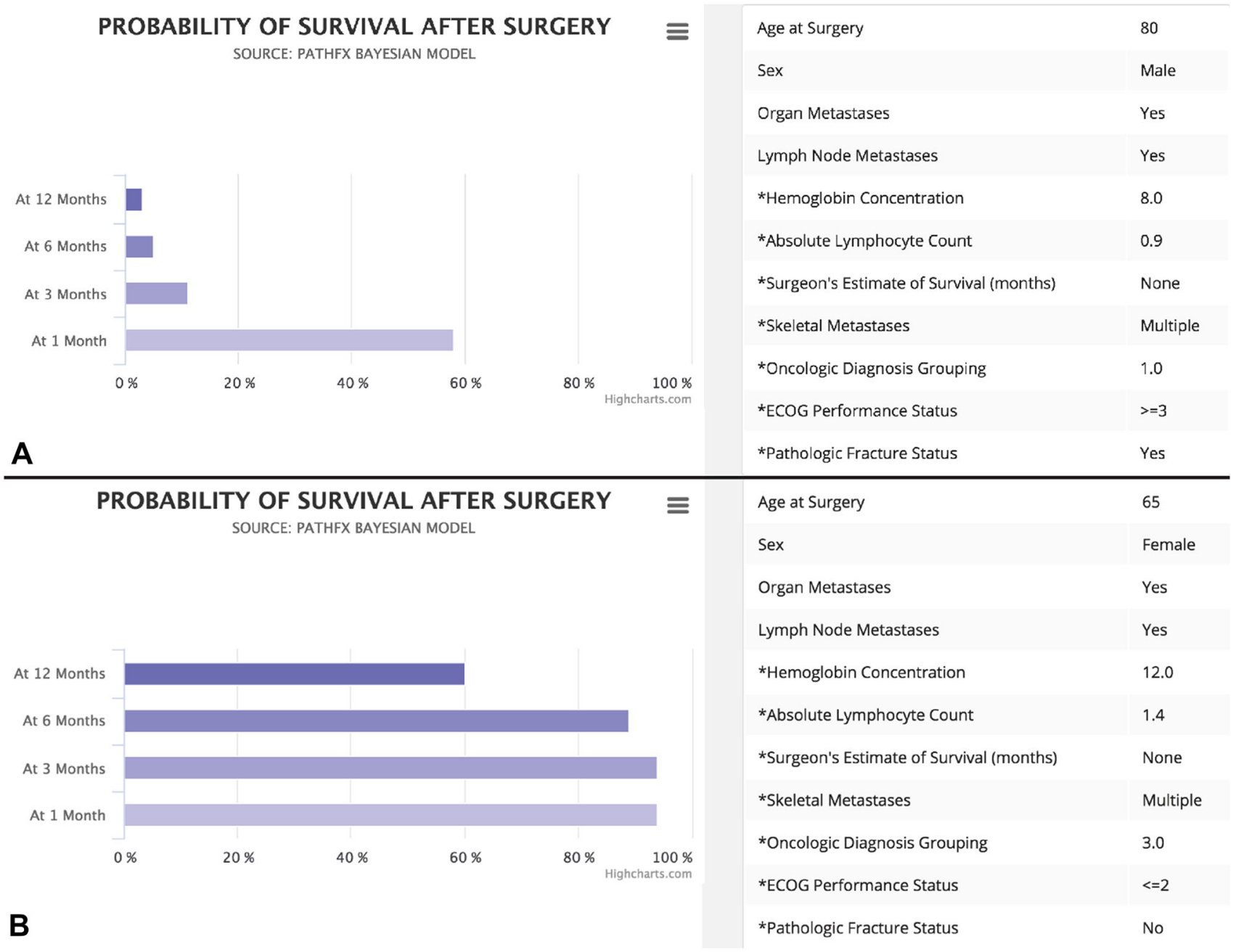

Fig. 5A-B This figure from www.pathfx.com shows two typical patient scenarios encountered by surgeons who treat metastatic bone disease. The patient characteristics and PATHFx inputs are shown on the right, and the individualized estimates of survival are displayed as horizontal bar graphs on the left. (A) The first graph shows a very poor survival profile that may help provide surgeons and families with objective information to choose palliative therapy, or in some cases, less-invasive means of surgical stabilization. (B) However, more favorable survival estimates can be used to justify the use of more durable, complicated, and expensive orthopaedic implants, such as conventional, or "tumor" prostheses. ECOG = Eastern Cooperative Oncology Group. 
apply the same level of scrutiny and healthy skepticism as they do for the implants they select, and the literature they read. The techniques used to establish discriminatory ability, and net benefit based on external validation, are considered to be the minimum statistical requirements for would-be clinical decision support tools. As such, these results apply to patients similar to those represented by the United States and Scandinavian populations who were treated at oncologic referral centers in a multidisciplinary fashion. If PATHFx is to be used in other settings, including international centers with differing patient populations and treatment philosophies, additional external validation is recommended and currently is underway.

When approaching patients with metastatic bone disease, surgeons base treatment decisions on various factors, including the location and physiology of the lesion, the desired mechanical properties of the implant, the patient's and family's wishes, and perhaps most importantly, how long the patient is likely to live. For instance, an 80-yearold male with multiple skeletal metastases, an impending pathologic femur fracture attributable to lung cancer, poor ECOG performance status $(\geq 3)$, organ and lymph node metastases, hemoglobin of $8 \mathrm{~g} / \mathrm{dL}$ on admission, and an absolute lymphocyte count of $0.9 \mathrm{~K} / \mathrm{mL}$ has a very unfavorable survival profile (Fig. 5A), which may help justify palliative measures, rather than more-complicated and expensive surgical options. However, a 65-year-old female with multiple skeletal metastases and an impending pathologic femur fracture attributable to estrogen receptorpositive breast cancer, organ metastases, lymph node metastases, a hemoglobin of $12 \mathrm{~g} / \mathrm{dL}$ on admission, and an absolute lymphocyte count of $1.4 \mathrm{~K} / \mathrm{mL}$ has a much more favorable survival profile (Fig. 5B), which we believe helps justify the need for more-durable reconstructive options.

On external validation, discriminatory ability of the 1 - and 6-month models was similar to those previously studied $[6,11]$. Specifically, the 3- month and 12-month models contained in PATHFx showed AUCs of 0.79 and 0.76, respectively, when confronted with this external validation data set and DCA showed that both of these models were suitable for use in the Scandinavian patient population [6]. The results of the current study show similar discriminatory ability of 0.76 (95\% CI, 0.72-0.80) and 0.76 (95\% CI, 0.730.79 ), for the 1- and 6-month models, respectively, and favorable DCA indicating that both models could be used rather than assume all patients, or none, would survive greater than 1 or 6 months, respectively.

One disadvantage of the PATHFx models has been an inability to compare them with other published means of estimating survival in patients with skeletal metastases. For instance, the method of Tokuhashi et al. [13], a widely used means to guide surgical decision-making in patients with symptomatic spine metastases, classifies estimated postoperative survival using 6 and 12 months. Before now, this method could not be compared with the PATHFx models, which previously contained only 3 - and 12-month models. In addition, the method of Katagiri et al. [9] generates short-, intermediate-, and long-term survival estimates using a scoring system. Although future work is necessary to determine whether the methods described by Tokuhashi et al. [13], Katagiri et al. [9], or PATHFx are better suited for clinical use, the techniques used to establish discriminatory ability, and net benefit of this article, considered to be the minimum statistical requirements for would-be clinical decision support tools, may be used to answer this question.

The Bayesian Belief Network appears well suited for estimating 1- and 6-month survival in this patient population. Measures of discriminatory ability and decision analysis confirm that both models are suitable for clinical use and we upgraded www.pathfx.org to include the algorithms described in this study. Surgeons may now use a web browser to generate survival estimates at $1,3,6$, and 12 months after surgery, at no cost. Although we plan further external validation studies in surgical and nonsurgical patients, we believe these objective estimates may be used not only to guide treatment, but also as a risk stratification method to support future clinical trials involving patients with metastatic bone disease.

Acknowledgments We thank Liz Silvius MS (DecisionQ Inc, Washington, DC, USA), and John Tra PhD (Department of Defense Osseointegration Program) for helpful data assembly and insight into Bayesian statistics.

\section{References}

1. Collins GS, Reitsma JB, Altman DG, Moons KG. Transparent reporting of a multivariable prediction model for individual prognosis or diagnosis (TRIPOD): the TRIPOD statement. $B M J$. 2015;350:g7594.

2. Cook NR. Use and misuse of the receiver operating characteristic curve in risk prediction. Circulation. 2007;115:928-935.

3. Forsberg JA. Suggested Guidelines. In Wedin R, Bauer H, and Weidenhielm L, eds. Turning Data Into Decisions. Stockholm, Sweden, Karolinska University Press, 2015: 56-57.

4. Forsberg JA, Eberhardt J, Boland PJ, Wedin R, Healey JH. Estimating survival in patients with operable skeletal metastases: an application of a bayesian belief network. PLOS ONE. 2011;6:e19956. doi:10.1371/journal.pone.0019956.

5. Forsberg JA, Sjoberg D, Chen QR, Vickers A, Healey JH. Treating metastatic disease: which survival model is best suited for the clinic? Clin Orthop Relat Res. 2013;471:843-850.

6. Forsberg JA, Wedin R, Bauer HC, Hansen BH, Laitinen M, Trovik CS, Keller JØ, Boland PJ, Healey JH. External validation of the Bayesian Estimated Tools for Survival (BETS) models in patients with surgically treated skeletal metastases. BMC Cancer. 2012;12:493 
7. Glare P, Virik K, Jones M, Hudson M, Eychmuller S, Simes J, Christakis N. A systematic review of physicians' survival predictions in terminally ill cancer patients. BMJ. 2003;327:195-198.

8. Hartsell WF, Desilvio M, Bruner DW, Scarantino C, Ivker R, Roach M 3rd, Suh J, Demas WF, Movsas B, Petersen IA, Konski AA. Can physicians accurately predict survival time in patients with metastatic cancer? Analysis of RTOG 97-14. J Palliat Med. 2008;11:723-728.

9. Katagiri H, Takahashi M, Wakai K, Sugiura H, Kataoka T, Nakanishi K. Prognostic factors and a scoring system for patients with skeletal metastasis. J Bone Joint Surg Br. 2005;87:698-703.

10. Mavrogenis AF, Angelini A, Vottis C, Pala E, Calabrò T, Papagelopoulos PJ, Ruggieri P. Modern palliative treatments for metastatic bone disease: awareness of advantages, disadvantages, and guidance. Clin J Pain. 2016;32:337-350.

11. Piccioli A, Spinelli MS, Forsberg JA, Wedin R, Healey JH, Ippolito V, Daolio PA, Ruggieri P, Maccauro G, Gasbarrini A,
Biagini R, Piana R, Fazioli F, Luzzati A, Di Martino A, Nicolosi F, Camnasio F, Rosa MA, Campanacci DA, Denaro V, Capanna R. How do we estimate survival? External validation of a tool for survival estimation in patients with metastatic bone disease: decision analysis and comparison of three international patient populations. BMC Cancer. 2015;15:424.

12. Steensma M, Healey JH. Trends in the surgical treatment of pathologic proximal femur fractures among Musculoskeletal Tumor Society members. Clin Orthop Relat Res. 2013;471:2000 2006.

13. Tokuhashi Y, Matsuzaki H, Oda H, Oshima M, Ryu J. A revised scoring system for preoperative evaluation of metastatic spine tumor prognosis. Spine (Phila Pa 1976). 2005;30:2186-2191.

14. Vickers AJ, Elkin EB. Decision curve analysis: a novel method for evaluating prediction models. Med Decis Making. 2006;26:565-574. 